\title{
The emerging role of serum zinc in motor disability and radiological findings in patients with multiple sclerosis
}

\author{
Mohammed I. Oraby', Mona Hussein ${ }^{1 *}$, Rehab Abd Elkareem² and Eman Elfar ${ }^{3}$
}

\begin{abstract}
Background: The role of serum Zinc in the pathogenesis of relapsing remittent multiple sclerosis (RRMS) and its impact on motor disability and radiological findings remains a matter of debate.

Objectives: To assess the level of serum Zinc in patients with RRMS at time of relapse and during remission and to correlate serum zinc with degree of disability, fatigue, response to disease modifying drugs, and magnetic resonance imaging (MRI) findings.

Subjects and methods: A case-control study was carried out on 75 subjects divided into three groups, 25 patients diagnosed as RRMS during relapse, 25 patients during remission, and 25 healthy controls. All included patients were subjected to neurological assessment including Expanded Disability Status Scale (EDSS), Modified fatigue impact scale (MFIS), and Modified RIO score (MRS). Zinc serum level was measured for all patients and controls using direct colorimetric test method. Brain and spine MRI were done for all included patients.

Results: MS patients in relapse and during remission were found to have significantly lower zinc level than the control group ( $P$ value $=0.001$ and $<0.001$ respectively). There was a statistically significant negative correlation between serum zinc level and disease duration, number of relapses in the last 2 years, total number of relapses, EDSS before and after pulse steroids, MRS, MFIS, and MRI lesion load ( $P$ value $=0.017,0.037,0.001,0.001,0.004,0.005$, and 0.001 , respectively).

Conclusion: Serum zinc level is significantly lower in patients with MS than healthy controls. It is negatively correlated with disease duration, number of relapses, motor disability, fatigue, and MRI lesion load.
\end{abstract}

Keywords: Multiple sclerosis, Zinc, EDSS, MFIS, MRS

\section{Introduction}

Multiple sclerosis (MS) is the most common autoimmune disease of the central nervous system (CNS) and one of the main causes of neurological disability in young adults. The prevalence of MS varies between 2 and 160 per 100, 000 according to the geographical area [1]. The etiology of the disease is not fully understood, though all the proposed theories point to the interplay between genetic and environmental risk factors [2]. Major histocompatibility complex with HLA-DRB1 showed the strongest genetic predisposition to MS [3]. Many genes have been proposed as well, $\mathrm{Zn}$-ion binding genes which are involved in

\footnotetext{
* Correspondence: mona.neuro@yahoo.com

${ }^{1}$ Department of Neurology, Beni-Suef University, Beni-Suef, Egypt

Full list of author information is available at the end of the article
}

interferon- $\gamma$ (IFN- $\gamma$ ) expression can predict the clinical outcome in relapsing remitting multiple sclerosis (RRMS) patients with high accuracy [4].

Zinc is an essential trace element, required by a considerable number of enzymes, transcription factors, and proteins [5, 6]. Actually, Zn deficiency suppress several immune system functions including T-helper cell function, cytotoxic $\mathrm{T}$ cell activity, peripheral $\mathrm{T}$ cell count, natural killer (NK) cells, neutrophil, and macrophage functions [7].

The brain has an abundant amount of $\mathrm{Zn}$ which is mainly found in the presynaptic vesicles. Efficient $\mathrm{Zn}$ homeostasis in neural cells is of great importance; deficiency of $\mathrm{Zn}$ can induce apoptosis while high level of $\mathrm{Zn}$ is reported to be neurotoxic $[8,9]$. 
To what extent zinc deficiency could play a critical role in the pathogenesis of multiple sclerosis and therefore affect disease progression, clinical outcome and disability status remain a matter of debate.

\section{Aim of the work}

The aim of this study was to assess serum Zinc level in patients with relapsing remittent multiple sclerosis at time of relapse and during remission and to correlate serum zinc with degree of disability, fatigue, response to disease modifying drugs, and magnetic resonance imaging (MRI) findings.

\section{Subjects and methods}

This is a case-control study which included 75 subjects divided into three groups, 25 patients diagnosed with relapsing remitting multiple sclerosis (during relapse), 25 patients diagnosed with relapsing remitting multiple sclerosis (during remission), and 25 age- and sex-matched healthy controls. The study was approved by the ethical committee in Faculty of medicine, Beni-Suef University (FWA00015574in 9th of October 2018), and all participants in this study signed a written informed consent.

Patients were diagnosed as relapsing remitting multiple sclerosis (RRMS) according to the International Panel on Diagnosis of Multiple Sclerosis "McDonald's criteria 2017" [10] (25 patients during relapse and 25 patients during remission). Relapse was defined by the appearance of new neurological symptoms or worsening of pre-existing neurological symptoms lasting at least $24 \mathrm{~h}$ in the absence of fever or infection in a patient who was neurologically stable or improving for the previous 30 days accompanied by objective changes on neurological examination (worsening on the Expanded Disability Status Scale (EDSS) of 0.5 point or a worsening by one point on the pyramidal, cerebellar, brainstem, or visual functional system scores). Age of the included patients ranged between 15 and 45 years.

The following patients were excluded from the study: patients with history of any other autoimmune disease, cerebrovascular disease, malignancy, hypertension, diabetes mellitus, any infectious or inflammatory diseases in the previous month, or pregnant females.

All patients included in the study were subjected to the following:

\section{Neurological assessment}

Expanded Disability Status Scale (EDSS): a scale used for assessment of the neurological disability status of MS patients. The functional systems included are pyramidal, brain stem, cerebellar, sensory, bowel and bladder, cerebral and visual. Its score ranged from 0 (normal) to 10 (death due to MS) [11].
Modified fatigue impact scale (MFIS): the modified version of the 40-item Fatigue Impact Scale (FIS), which was used to evaluate the effect of fatigue on quality of life in patients with MS and can be used in patients with other chronic diseases. The full-length MFIS consists of 21 items; 10 "cognitive" items, 9 "physical" items, and 2 "psychosocial" items. Higher scores indicate a greater impact on patient's quality of life, and the maximum possible score is 84 . The time needed to complete the scale is approximately $5-10 \mathrm{~min}$ [12].

Modified RIO score (MRS): a 0 to 3 scale which evaluates the response of MS patients to disease modifying therapy. It is obtained by a combination of MRI and relapse criteria. (i) MRI criterion 1 point for patients with $>4$ new T2 lesions; (ii) relapse criterion 1 point for patients experiencing 1 relapse and 2 points for patients experiencing $\geq 2$ relapses [13].

\section{Laboratory assessment}

Serum level of zinc was measured for all patients and controls included in this study using direct colorimetric test method (Reactivos GPL, Barcelona, Spain). This was applied at clinical pathology department, Beni-Suef University hospital on a semi-automated photometer (ELITechGroup VITAL, Dieren-The Netherlands). Five milliliters of whole blood was collected from both patients and controls into sterile plain tube and centrifuged for $10-15 \mathrm{~min}$ at 3000 r.p.m. Supernatant clear sera were collected, and Zinc was immediately measured at wave length $578 \mathrm{~nm}$ at $37^{\circ} \mathrm{C}$ (range of normal value $=68-107 \mu \mathrm{g} / \mathrm{dL}$ )

\section{Radiological assessment}

Magnetic resonance imaging (MRI) on brain, cervical, and dorsal segments of the spinal cord were carried out for all patients included in this study to detect site, size, and number of MS plaques and to exclude other structural lesions. The scan was done by a 1.5 Tesla Siemens scanner, Germany. The following sequences were used: T1-weighted images (axial, sagittal), T2-weighted images (axial, coronal), fluid-attenuated inversion recovery (FLAIR), and gadolinium-enhanced T1-weighted axial and sagittal images for patients during relapse.

\section{Statistical methods}

The data were coded and entered using the statistical package for social science (SPSS) version 18 (SPSS Inc. Released 2009. PASW Statistics for Windows, Version 18.0. Chicago: SPSS Inc.). Student $t$ test was used for comparison between means of quantitative variables in patients and control group. Chi-square test was used for comparison between categorical data in patients and control group. The one way analysis of variance (ANOVA) and post hoc analysis (Bonferonni test) were used to assess statistical differences between quantitative data inpatients 
Table 1 Demographics of MS patients and control group

\begin{tabular}{|c|c|c|c|c|c|}
\hline \multirow{2}{*}{\multicolumn{2}{|c|}{$\overline{\text { Age [mean (SD)] }}$}} & Patients in relapse $(n=25)$ & Patients in remission $(n=25)$ & Controls $(n=25)$ & $P$ value \\
\hline & & $30.88(9.01)$ & $32.28(8.07)$ & $28.28(7.08)$ & 0.214 \\
\hline \multirow[t]{2}{*}{ Sex } & Male $[n(\%)]$ & $4(16 \%)$ & $9(36 \%)$ & $11(44 \%)$ & 0.092 \\
\hline & Female $[n(\%)]$ & $21(84 \%)$ & $16(64 \%)$ & $14(56 \%)$ & \\
\hline
\end{tabular}

$P$ value $\geq 0.05$ (non-significant)

in relapse, patients in remission, and control group. The Pearson correlation coefficient $(r)$ was used to describe the degree of relationship between serum zinc level and disease duration, number of relapses in the last 2 years, total number of relapses, EDSS before and after pulse steroids, MRS, MFIS, and MRI lesion load. $P$ value equal to or less than 0.05 was considered significant.

\section{Results}

The mean age for patients in relapse was $(30.88 \pm 9.01)$ years, for patients in remission was $(32.28 \pm 8.07)$ years, and for controls was $(28.28 \pm 7.08)$ years. Regarding sex, $16 \%(n=4)$ of patients in relapse were males and $84 \%(n=$ $21)$ were females, and $36 \%(n=9)$ of patients in remission were males and $64 \%(n=16)$ were females. As for controls, $44 \%(n=11)$ were males and $56 \%(n=14)$ were females $(p$ $=0.47)$. There was no statistically significant difference between patients and controls in either age $(P$ value $=0.214)$ or sex $(P$ value $=0.092)($ Table 1$)$.

Regarding clinical and radiological characteristics of MS patients, there was no statistically significant difference between patients in relapse and patients in remission in either disease duration, number of relapses in the last 2 years, total number of relapses, EDSS, MRS, MFIS, or MRI lesion load (Table 2).
Regarding serum zinc level, patients in relapse were found to have significantly lower mean values $(65.29 \pm$ $16.92) \mu \mathrm{g} / \mathrm{dL}$ than the control group $(82.15 \pm 15.496) \mu \mathrm{g} /$ $\mathrm{dL}(P$ value $=0.001)$. Patients in remission were also found to have significantly lower mean values $(67.97 \pm 15.28)$ $\mu \mathrm{g} / \mathrm{dL}$ than the control group $(82.15 \pm 15.496) \mu \mathrm{g} / \mathrm{dL}(P$ value $<0.001)$. There was no statistically significant difference between patients in relapse and patients in remission in serum zinc level $(P$ value $=0.56)($ Table 3$)$.

There was a statistically significant negative correlation between serum zinc level and disease duration, number of relapses in the last 2 years, total number of relapses, EDSS before and after pulse steroids, MRS, MFIS, and MRI lesion load (Table 4).

\section{Discussion}

In the last few years, serum zinc levels was thoroughly investigated in patients with multiple sclerosis to understand its potential role in pathogenesis of MS. Lower serum zinc levels were found to be associated with higher disease disability[14].

The aim of this work was to evaluate serum Zinc level in patients with relapsing remittent multiple sclerosis at time of relapse and during remission and to correlate

Table 2 Clinical and radiological characteristics of MS patients

\begin{tabular}{|c|c|c|c|c|}
\hline & & Patients in relapse $(n=25)$ & Patients in remission $(n=25)$ & $P$ value \\
\hline \multicolumn{2}{|c|}{ Disease duration [mean(SD)] } & $4.6800(4.69)$ & $5.52(5.06)$ & 0.545 \\
\hline \multicolumn{2}{|c|}{ Number of relapses in the last 2 years [mean (SD)] } & $1.52(0.59)$ & $1.36(0.7)$ & 0.385 \\
\hline \multicolumn{2}{|c|}{ Total number of relapses [mean (SD)] } & $2.6(1.55)$ & $3.28(2.05)$ & 0.193 \\
\hline \multirow[t]{5}{*}{ DMDs } & Monthly methylprednisolone [n (\%)] & $18(72.0 \%)$ & $13(52.0 \%)$ & \multirow[t]{5}{*}{$0.01^{*}$} \\
\hline & Interferon beta-1a (Avonex) $[n(\%)]$ & $0(0.0 \%)$ & $7(28.0 \%)$ & \\
\hline & Interferon beta-1b (Betaferon) [n (\%)] & $5(20.0 \%)$ & $1(4.0 \%)$ & \\
\hline & Interferon beta-1a (Rebif) $[n(\%)]$ & $1(4.0 \%)$ & $4(16.0 \%)$ & \\
\hline & Azathioprine $[n(\%)]$ & $1(4.0 \%)$ & $0(0.0 \%)$ & \\
\hline \multicolumn{2}{|c|}{ EDSS before pulse steroids [mean (SD)] } & $4.08(2.06)$ & - & - \\
\hline \multicolumn{2}{|c|}{ EDSS after pulse steroids [mean (SD)] } & $2.9400(1.72)$ & $2.88(1.29)$ & 0.889 \\
\hline \multicolumn{2}{|c|}{ MRS [mean (SD)] } & $0.3200(0.63)$ & $0.2(0.5)$ & 0.458 \\
\hline \multicolumn{2}{|c|}{ MFIS[mean (SD)] } & $7.24(4.81)$ & $6.56(4.19)$ & 0.596 \\
\hline \multicolumn{2}{|c|}{ MRI lesion load [mean (SD)] } & $11.0400(6.29)$ & $11.0800(5.35)$ & 0.980 \\
\hline
\end{tabular}

DMDs disease modifying drugs, EDSS Expanded Disability Status Scale, MRS modified RIO score, MFIS modified fatigue impact scale, MRI magnetic resonance imaging

${ }^{*} P$ value $<0.05$ (significant) 
Table 3 Serum zinc level in MS patients and control group

\begin{tabular}{|c|c|c|c|c|}
\hline & Patients in relapse $(n=25)$ & Patients in remission $(n=25)$ & Controls $(n=25)$ & $P$ value \\
\hline Serum zinc level in $\mu \mathrm{g} / \mathrm{dL}$ [mean (SD)] & $65.29(16.92)$ & $67.97(15.28)$ & $82.15(15.496)$ & $0.001^{*}$ \\
\hline
\end{tabular}

serum zinc with degree of disability, fatigue, response to disease modifying drugs, and MRI findings.

In the present study, MS patients in relapse and remission were found to have significantly lower serum Zinc level compared to controls. There was a statistically significant negative correlation between serum zinc level and disease duration, number of relapses in the last 2 years, total number of relapses, EDSS before and after pulse steroids, MRS, MFIS, and MRI lesion load.

Similar to our findings, Pawlitzki and colleagues found that MS patients had a significantly lower concentrations of zinc compared to healthy controls but there was no significant relationship between serum zinc and either disease duration, median number of relapses, annual relapse rate, or EDSS [15].

Another study carried out by Ho and colleagues reported that serum levels of $\mathrm{Zn}$ significantly decrease during relapse and increase during remission [16].

On the other hand, Geeta and colleagues found no difference in serum zinc levels between MS patients and control groups [17]. Additionally, Sedighidid and colleagues did not find a significant difference between serum zinc levels in MS patients and controls [18]. The same findings were also obtained by Mellow and colleagues who compared zinc level in patients with MSto control group [19].

A recent meta-analysis carried out by Bredholt and Frederiksen included 13 studies evaluating plasma or serum $\mathrm{Zn}$ levels in MS patients. The investigators found that MS patients had a statistically significant reduction in overall plasma or serum $\mathrm{Zn}$ levels, with few studies

Table 4 Correlation between serum Zinc level and clinical and radiological characteristics of MS

\begin{tabular}{lll}
\hline & \multicolumn{2}{l}{ Serum zinc level } \\
\cline { 2 - 3 } & $(r)$ coefficient & $P$ value \\
\hline Disease duration & -0.335 & $0.017^{*}$ \\
Number of relapses in the last 2 years & -0.296 & $0.037^{*}$ \\
Total number of relapses & -0.466 & $0.001^{*}$ \\
EDSS before pulse steroids & -0.771 & $<0.001^{*}$ \\
EDSS after pulse steroids & -0.785 & $<0.001^{*}$ \\
MRS & -0.403 & $0.004^{*}$ \\
MFIS & -0.394 & $0.005^{*}$ \\
MRI lesion load & -0.790 & $<0.001^{*}$ \\
\hline
\end{tabular}

EDSS Expanded Disability Status Scale, MRS modified RIO score, MFIS Modified fatigue Impact Scale, MRI magnetic resonance imaging

${ }^{*} P$ value $<0.05$ (significant) reporting either increase or no difference in plasma or serum $\mathrm{Zn}$ levels [14].

Zinc plays an important role in suppression of potentially harmful immune reactions against $\mathrm{T}$ lymphocytes, and predisposing inflammatory responses of MS. Zn also has an antioxidant effect protecting myelin and cell membranes [20].

During active disease stages, upregulation of zincdependent matrix metalloproteinases occurs which in turn leads to lower zinc levels in MS patients [21]. Zinc deficiency is also reported to play an important role in inducing an imbalance between Th1 and Th2 functions [22], in addition to its role in prohibiting downregulation of Th17 lymphocytes [23]. These changes have been proposed to be major mechanisms in the pathogenesis of MS [24].

Also, zinc was reported to be involved in releasing tumor necrosis factor alpha (TNF $\alpha$ ), which plays an important role in immune system activation. Consequently, even a mild $\mathrm{Zn}$ deficiency can impair the immune system functions $[25,26]$.

The results of studies which assessed the effect of $\mathrm{Zn}$ supplementation revealed a reduction in proliferation and activation of $\mathrm{T}$ cell following $\mathrm{Zn}$ supplementation thereby considered a potential therapeutic approach to MS [27]. On the other hand, a recent study revealed that neurological signs did not improve in patients with MS after $\mathrm{Zn}$ supplementation for 3 months compared to the placebo group [28]. An interesting study carried out by Choi and colleagues examined the effect of redistributing $\mathrm{Zn}$ in Experimental Autoimmune Encephalitis (EAE) in an animal model of MS using Clioquinol (a Zincor Copper chelator) [29]. The study revealed promising results regarding suppression of demyelination, inhibition of BBB disruption, reduced Matrix metalloproteinase- 9 activation, reduced infiltration of encephalitogenic immune cells, and reduced clinical score of EAE following treatment with Clioquinol.

\section{Conclusion}

At the end of the study, we conclude that serum Zinc level is significantly lower in patients with multiple sclerosis than healthy controls. It is negatively correlated with disease duration, number of relapses, motor disability, fatigue, and MRI lesion load.

The limitation of this work is the relatively small number of patients due to financial issues and the limitation of resources. 
Further studies should be conducted on a larger number of MS patients to estimate the effect of zinc supplementation on motor disability, fatigability, and number of relapses.

\begin{abstract}
Abbreviations
CNS: Central nervous system; DMDs: Disease modifying drugs;

EAE: Experimental Autoimmune Encephalitis; EDSS: Expanded Disability Status Scale; FIS: Fatigue Impact Scale; FLAIR: Fluid attenuated inversion recovery; IFN- $\gamma$ : Interferon- $\gamma ;$ MFIS: Modified fatigue impact scale; MRI: Magnetic resonance imaging; MRS: Modified RIO score; MS: Multiple sclerosis; NK: Natural killer; RRMS: Relapsing remitting MS; TNFa: Tumor necrosis factor alpha
\end{abstract}

\section{Acknowledgements}

Not applicable

\section{Authors' contribution}

$\mathrm{MO}$ participated in the study design, sequence alignment, and collection and analysis of data and helped to draft the manuscript. $\mathrm{MH}$ participated in the study design and helped to draft the manuscript. RA participated in the study design and performed the laboratory work. EE participated in the study design, sequence alignment, and collection and analysis of data. All authors read and approved the final manuscript

\section{Funding}

Authors did not receive any funding for this work.

\section{Availability of data and materials}

The datasets used and/or analyzed during the current study are available from the corresponding author on reasonable request with permission of Faculty of Medicine, Beni-Suef University, Egypt.

\section{Ethics approval and consent to participate}

A written informed consent was obtained from each participant in this study or from one of his family members, and the study was approved by local ethical committee in Faculty of medicine, Beni-Suef University (the number is FWA00015574, and the date is 9th of October 2018).

\section{Consent for publication}

Not applicable

\section{Competing interests}

The authors declare that they have no competing interests

\section{Author details}

'Department of Neurology, Beni-Suef University, Beni-Suef, Egypt.

${ }^{2}$ Department of Clinical and Chemical Pathology, Beni-suef University, Beni-Suef 62511, Egypt. ${ }^{3}$ Public health and community medicine lecturer, Cairo University, Cairo, Egypt.

Received: 5 October 2018 Accepted: 21 August 2019

Published online: 05 September 2019

\section{References}

1. Pugliatti M, Sotgiu S, Rosati G. The worldwide prevalence of multiple sclerosis. ClinNeurolNeurosurg. 2002;104(3):182-91.

2. Sadovnick AD, Ebers GC. Epidemiology of multiple sclerosis: a critical overview. Can J Neurol Sci. 1993;20(1):17-29.

3. Patsopoulos NA, Barcellos LF, Hintzen RQ, Schaefer C, van Duijn CM, Noble JA, et al. Fine-mapping the genetic association of the major histocompatibility complex in multiple sclerosis: HLA and non-HLA effects. PLoS Genet. 2013;9(11):e1003926

4. Achiron A, Gurevich M, Snir Y, Segal E, Mandel M. Zinc-ion binding and cytokine activity regulation pathways predicts outcome in relapsing-remitting multiple sclerosis. ClinExplmmunol. 2007;149(2): 235-42.

5. Maywald M, Wessels I, Rink L. Zinc signals and immunity. Int J Mol Sci. 2017;18:2222.
6. Vallee BL, Falchuk KH. The biochemical basis of zinc physiology. Physiol Rev. 1993;73: 79-118.

7. Rink L, Gabriel P. Zinc and the immune system. The Proceedings of the Nutrition Society. 2000;59:541-52.

8. Yokoyama M, Koh J, Choi DW. Brief exposure to zinc is toxic to cortical neurons. Neuroscience Letters. 1986:71:351-5.

9. Adamo AM, Zago MP, Mackenzie GG, Aimo L, Keen CL, Keenan A, et al. The role of zinc in the modulation of neuronal proliferation and apoptosis. Neurotox Res. 2010; 17(1):1-14.

10. Thompson AJ, Banwell BL, Barkhof F, Carroll WM, Coetzee T, Comi G, et al. Diagnosis of multiple sclerosis: 2017 revisions of the McDonald criteria. Lancet Neurol. 2018;17: $162-73$.

11. Kurtzke JF. Rating neurologic impairment in multiple sclerosis: an expanded disability status scale (EDSS). Neurology. 1983;33:1444-52.

12. Fisk JD, Ritvo PG, Ross L, Haase DA, Marrie TJ, Schlech WF. Measuring the functional impact of fatigue: initial validation of the fatigue impact scale. Clin Infect Dis. 1994; 18Suppl 1:S79-83.

13. Sormani MP, Rio J, Tintore M, Signori A, Li D, Cornelisse P, et al. Scoring treatment response in patients with relapsing multiple sclerosis. MultScler. 2013;19:605-12.

14. Bredholt M, Frederiksen JL. Zinc in multiple sclerosis: a systematic review and metaanalysis. ASN Neuro. 2016;8.

15. Pawlitzki M, Uebelhör J, Sweeney-Reed C, Stephanik H, Hoffmann J, Lux A, et al. Lower serum zinc levels in patients with multiple sclerosis compared to healthy controls. Nutrients. 2018;10(8):967.

16. Ho SY, Catalanotto FA, Lisak RP, Dore-Duffy P. Zinc in multiple sclerosis. Il: correlation with disease activity and elevated plasma membrane-bound zinc in enythrocytes from patients with multiple sclerosis. Ann Neurol. 1986;20:712-5.

17. Geeta SMR, Sanne AM, Jacques DK Dietary patterns in clinical subtypes of multiple sclerosis: an exploratory study. Nutr J. 2009:8:36.

18. Sedighi B, Ebrahimi HA, Haghdoost AA, Abotorabi M. Comparison of serum levels of copper and zinc among multiple sclerosis patients and control group. Iran I Neurol. 2013;12(4):125-8.

19. Melø TM, Larsen C, White LR, Aasly J, Sjøbakk TE, Flaten TP, et al. Manganese, copper, and zinc in cerebrospinal fluid from patients with multiple sclerosis. Biol Trace Elem Res. 2003;93(1-3):1-8.

20. Smith DK, Feldman EB, Feldman DS. Trace element status in multiple sclerosis. Am J ClinNutr. 1989:50(1):136-40.

21. Kanesaka T, Mori M, Hattori T, Oki T, Kuwabara S. Serum matrix metalloproteinase-3 levels correlate with disease activity in relapsing-remitting multiple sclerosis. J NeurolNeurosurg Psychiatry. 2006;77:185-8.

22. Prasad AS. Effects of zinc deficiency on Th1 and Th2 cytokine shifts. J Infect Dis. 2000;182:562-8.

23. Kitabayashi C, Fukada T, Kanamoto M, Ohashi W, Hojyo S, Atsumi T, et al. Zinc suppresses Th17 development via inhibition of STAT3 activation. Intlmmunol. 2010; 22(5):375-86.

24. Rostami A, Ciric B. Role of Th17 cells in the pathogenesis of CNS inflammatory demyelination. J Neurol Sci. 2013;333:76-87.

25. Wagner S, Breyholz HJ, Faust A, Höltke C, Levkau B, Schober O, et al. Molecular imaging of matrix metalloproteinases in vivo using small molecule inhibitors for SPECT and PET. Curr Med Chem. 2006;13(23):2819-38.

26. Chandler S, Miller KM, Clements JM, Lury J, Corkill D, Anthony DC, et al. Matrix metalloproteinases, tumor necrosis factor and multiple sclerosis: an ovenview. J Neuroimmunol. 1997;72(2):155-61

27. Stoye D, Schubert C, Goihl A, Guttek K, Reinhold A, Brocke S, et al. Zinc aspartate suppresses $T$ cell activation in vitro and relapsing experimental autoimmune encephalomyelitis in SJL/J mice. Biometals. 2012;25(3):529-39.

28. Salari S, Khomand P, Arasteh M, Yousefzamani B, Hassanzadeh K. Zinc sulphate: a reasonable choice for depression management in patients with multiple sclerosis: a randomized, double-blind, placebo-controlled clinical trial. Pharmacological Reports. 2015;67:606-9.

29. Choi BY, Jang BG, Kim JH, Seo JN, Wu G, Sohn M, et al. Copper/zinc chelation by clioquinol reduces spinal cord white matter damage and behavioral deficits in a murine MOG-induced multiple sclerosis model. Neurobiol Dis. 2013;54:382-91.

\section{Publisher's Note}

Springer Nature remains neutral with regard to jurisdictional claims in published maps and institutional affiliations. 